Louisiana State University

LSU Digital Commons

Faculty Publications

Department of Physics \& Astronomy

$12-2-2009$

\title{
Quantum forbidden-interval theorems for stochastic resonance
}

Mark M. Wilde

University of Southern California

Bart Kosko

University of Southern California

Follow this and additional works at: https://digitalcommons.Isu.edu/physics_astronomy_pubs

\section{Recommended Citation}

Wilde, M., \& Kosko, B. (2009). Quantum forbidden-interval theorems for stochastic resonance. Journal of Physics A: Mathematical and Theoretical, 42 (46) https://doi.org/10.1088/1751-8113/42/46/465309

This Article is brought to you for free and open access by the Department of Physics \& Astronomy at LSU Digital Commons. It has been accepted for inclusion in Faculty Publications by an authorized administrator of LSU Digital Commons. For more information, please contact ir@lsu.edu. 


\title{
Quantum Forbidden-Interval Theorems for Stochastic Resonance
}

\author{
Mark M. Wilde ${ }^{1,2 *}$ and Bart Kosko ${ }^{1}$ \\ ${ }^{1}$ Center for Quantum Information Science and Technology, Department of Electrical Engineering, \\ University of Southern California, Los Angeles, California 90089, USA and \\ ${ }^{2}$ Hearne Institute for Theoretical Physics, Department of Physics and Astronomy, \\ Louisiana State University, Baton Rouge, Louisiana 70803
}

(Dated: October 23, 2021; Received; Revised; Accepted; Published)

\begin{abstract}
We extend the classical forbidden-interval theorems for a stochastic-resonance noise benefit in a nonlinear system to a quantum-optical communication model and a continuous-variable quantum key distribution model. Each quantum forbidden-interval theorem gives a necessary and sufficient condition that determines whether stochastic resonance occurs in quantum communication of classical messages. The quantum theorems apply to any quantum noise source that has finite variance or that comes from the family of infinite-variance alpha-stable probability densities. Simulations show the noise benefits for the basic quantum communication model and the continuous-variable quantum key distribution model.
\end{abstract}

PACS numbers: 03.67.-a, 03.67.Hk, 42.50.Dv, 05.45.Vx, 05.45.-a

Keywords: stochastic resonance, quantum optics, alpha-stable noise, quantum communication

Stochastic resonance (SR) occurs in a nonlinear system when noise benefits the system [1, 2, [3, 4, [5]. SR can occur in both classical and quantum systems [4, 6] that use noise to help detect faint signals. The footprint of SR is a nonmonotonic curve that results when the system performance measure depends on the intensity of the noise source. Figure 1 shows such an SR surface for a quantum-optical communication system with both additive channel noise and squeezing noise. Mutual information measures the noise benefits in bits.

The classical SR forbidden-interval theorems give necessary and sufficient conditions for an SR noise benefit in terms of mutual information [7, 8] when the system nonlinear is a threshold. The noise benefit turns on whether the noise mean or location $a$ lies in an interval that depends on the threshold $\theta$ and the bipolar subthreshold signals $A$ and $-A$ : SR occurs if and only if $a \notin(\theta-A, \theta+A)$ where $-A<A<\theta$. This result holds for all finite-variance noise and all infinite-variance stable noise. But it guarantees only that some SR noise benefit occurs in the system for the given choice of parameters. SR stochastic learning algorithms [9] can then search for the optimal noise level.

This paper generalizes the classical forbidden-interval theorems to a quantum-optimal communication system that uses squeezed light [10, 11]. The corresponding quantum forbidden-interval theorems give similar necessary and sufficient conditions for a noise benefit but include the strength of light squeezing as a parameter. The quantum-optical system in Figure 1 produces SR because the noise mean is zero and so does not lie in the system's forbidden interval $(.5,2.7)$. We also show that modified versions of the quantum forbidden-interval theorems hold in continuous-variable quantum key dis-

*Electronic address: mark.wilde@usc.edu
Threshold $\theta=1.6, \quad$ Signal value $\alpha_{x}=1.1$

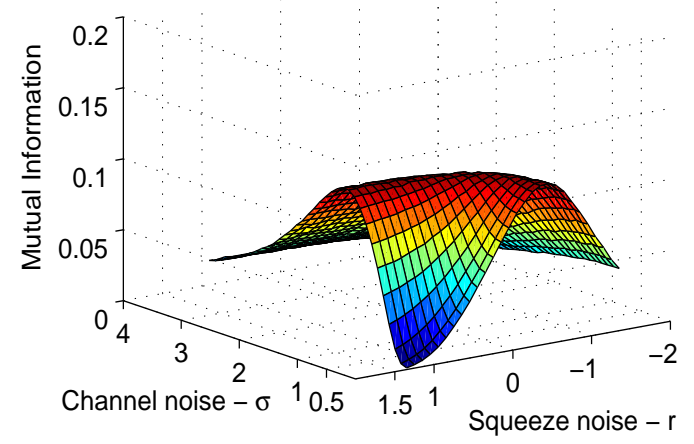

FIG. 1: (Color online) Stochastic resonance in the basic quantum-optical communication model with Gaussian noise. The sender Alice encodes coherent states with amplitude $A=1.1$. The receiver Bob decodes with threshold $\theta=1.6$. The graph shows the smoothed mutual information as a function of the standard deviation $\sigma$ of the quantum Gaussian noise and the squeezing strength $r$ for 100 simulation runs. Each run generated 10,000 input-output signal pairs to estimate the mutual information. The SR effect occurs because the channel noise mean $\mu=0$ and thus $\mu$ lies outside the forbidden interval $(.5,2.7)$.

tribution with thresholding [12, 13].

Model for Quantum-Optical Thresholding System -We first develop the basic quantum-optical communication protocol. The first quantum forbidden-interval theorem applies to this communication model. We present the sender Alice's operations for encoding information, the effect of the noisy quantum channel on the state that Alice transmits, and the receiver Bob's detection scheme.

Alice wants to encode a message bit $S$ using quantumoptical techniques. The protocol begins with Alice possessing a vacuum mode. We describe our model in the 
Heisenberg picture. Let $\hat{x}$ denote the position-quadrature operator of Alice's vacuum mode where $\hat{x}=\left(\hat{a}+\hat{a}^{\dagger}\right) / \sqrt{2}$ and $\hat{a}$ is the annihilation operator for her vacuum mode [11]. We consider only the position-quadrature operator's evolution. Her vacuum state collapses to a zeromean 1/2-variance Gaussian random variable $X$ if she measures it with an ideal position-quadrature homodyne detector. Suppose that Alice does not measure it. Suppose instead that she sends her mode through a positionquadrature squeezer. Suppose further that she can control the strength of squeezing with a squeezing parameter $r$. The position-quadrature squeezer is a unitary operator $\hat{S}(r) \equiv \exp \left\{r\left(\hat{a}^{2}-\left(\hat{a}^{\dagger}\right)^{2}\right)\right\}[11]$. Her operator $\hat{x}$ evolves under the squeezer as $\hat{S}^{\dagger}(r) \hat{x} \hat{S}(r)=\hat{x} e^{-r}$. She encodes the random message bit $S \in\{0,1\}$ by displacing her state by $\alpha \in \mathbb{C}$ if $S=1$ or by $-\alpha$ if $S=0$. The displacement is a unitary operator $\hat{D}(\alpha) \equiv$ $\exp \left\{\alpha \hat{a}^{\dagger}-\alpha^{*} \hat{a}\right\}$ [1]. Let $\alpha_{S}$ be the conditional displacement $\alpha_{S}=(-1)^{S+1} \alpha$. Her operator $\hat{x} e^{-r}$ evolves under the conditional displacement $\hat{D}\left(\alpha_{S}\right)$ as

$$
\hat{D}^{\dagger}\left(\alpha_{S}\right)\left(\hat{x} e^{-r}\right) \hat{D}\left(\alpha_{S}\right)=\hat{x} e^{-r}+(-1)^{S+1} \alpha_{x}
$$

where $\alpha_{x}=\operatorname{Re}\{\alpha\}$. This equality gives the Heisenbergpicture observable that corresponds to Alice's mode before she sends it over the noisy channel. The message bit $S$ appears as a displacement in (1).

Alice sends her mode to Bob over an additive noisy bosonic channel [14] that adds a random displacement $\nu \in \mathbb{C}$ to its input state. The channel randomly displaces any annihilation operator $\hat{a}$ as $\hat{D}^{\dagger}(\nu) \hat{a} \hat{D}(\nu)=$ $\hat{a}+\nu$. This is the quantum-channel analogue to a classical continuous additive noisy channel [15]. The term $\hat{x} e^{-r}+(-1)^{S+1} \alpha_{x}+\nu_{x}$ is the Heisenberg-picture positionquadrature observable that corresponds to the state that Bob receives after Alice sends her mode over the noisy channel. Random variable $\nu_{x}=\operatorname{Re}\{\nu\}$ and corresponds to the position-quadrature noise.

Bob detects the information that Alice encodes by performing position-quadrature homodyne detection with inefficient photodetectors. We model this non-ideal homodyne detection as a lossy transmission through a material with linear absorption [16]. A beamsplitter with transmittivity $\eta$ models the linear absorptive material. Then the Heisenberg-picture observable after the lossy beamsplitter is

$$
\sqrt{\eta}\left((-1)^{S+1} \alpha_{x}+\hat{x} e^{-r}+\nu_{x}\right)+\sqrt{1-\eta} \hat{x}_{H}
$$

where $\eta$ is the quantum efficiency of the homodyne detection and $\hat{x}_{H}$ is the position quadrature operator of an input vacuum mode. Bob measures the position quadrature observable and the state collapses to the random variable

$$
\sqrt{\eta}\left((-1)^{S+1} \alpha_{x}+X e^{-r}+\nu_{x}\right)+\sqrt{1-\eta} X_{H} .
$$

$X_{H}$ is a zero-mean $1 / 2$-variance Gaussian random variable that corresponds to the vacuum observable $\hat{x}_{H}$.
Random variables $X e^{-r}, \nu_{x}$, and $X_{H}$ are independent because random variable $X e^{-r}$ comes from the vacuum fluctuations of Alice's original mode, because $\nu_{x}$ is Bob's loss of knowledge due to the state's propagation through a noisy quantum channel, and because $X_{H}$ comes from the vacuum contributions of non-ideal position-quadrature homodyne detection. Let random variable $N$ sum all noise terms:

$$
N \equiv \sqrt{\eta}\left(X e^{-r}+\nu_{x}\right)+\sqrt{1-\eta} X_{H} .
$$

The density $p_{N}(n)$ of random variable $N$ is

$$
p_{N}(n)=\left(p_{\sqrt{\eta} X e^{-r}} * p_{\sqrt{\eta} \nu_{x}} * p_{\sqrt{1-\eta} X_{H}}\right)(n)
$$

where $p_{\sqrt{\eta} X e^{-r}}(n)$ is the density of a zero-mean $\eta e^{-2 r} / 2$ variance Gaussian random variable, $p_{\sqrt{\eta} \nu_{x}}(n)$ is the density of $\sqrt{\eta} \nu_{x}, p_{\sqrt{1-\eta} X_{H}}(n)$ is the density of a zero-mean $(1-\eta) / 2$-variance Gaussian random variable, and $*$ denotes convolution. The density $p_{N}(n)$ is a convolution because random variables $X e^{-r}, \nu_{x}$, and $X_{H}$ are independent. So Bob's received signal using (3) and (4) is $\sqrt{\eta}(-1)^{S+1} \alpha_{x}+N$. Bob thresholds the result of the non-ideal homodyne detection with a threshold $\theta$ to retrieve a random bit $Y$ where

$$
Y \equiv u\left(\sqrt{\eta}(-1)^{S+1} \alpha_{x}+N-\theta\right)
$$

and $u$ is the unit Heaviside step function defined as $u(x)=1$ if $x \geq 0$ and $u(x)=0$ if $x<0$. This final bit $Y$ that Bob detects should be the message bit $S$ that Alice first sent.

Quantum Alpha-Stable Noise - The noise random variable $\nu_{x}$ need not have a finite second moment or finite higher-order moments. Some researchers argue that quantum-optical noise arises from a large number of independent random effects and and that it is Gaussian because of the central limit theorem [17, 18]. But these random effects need not converge to a Gaussian random variable even though they converge to a random variable with a bell-curve density. The generalized central limit theorem states that all and only normalized stable random variables converge in distribution to a stable random variable [19]. So an impulsive quantum noise source may have a limiting alpha-stable density through aggregation or directly through transformation as when the Cauchy density arises from the tangent of uniform noise.

Alpha-stable noise models diverse physical phenomena such as impulsive interrupts in phone lines, underwater acoustics, low-frequency atmospheric signals, and gravitational fluctuations [20]. The parameter $\alpha$ (different from "coherent state" $\alpha$ ) lies in $(0,2]$ and parametrizes the thickness of the curve's tails. The curve's tail thickness increases as $\alpha$ decreases: $\alpha=1$ corresponds to the thick-tailed Cauchy random variable and $\alpha=2$ corresponds to the familiar thin-tailed Gaussian random variable. Parameter $\beta$ is a skewness parameter such that $\beta=0$ gives a symmetric density. Parameter $\gamma$ is a dispersion parameter that acts like the variance because it 
quantifies the spread or width of the alpha-stable density around its location parameter $a$.

Quantum Forbidden-Interval Theorem-Theorem 1 below shows that any finite-variance quantum noise or any infinite-variance alpha-stable noise produces the SR effect in our model. The theorem states that the SR effect occurs for finite-variance noise if and only if the noise mean $\mu_{\nu_{x}}$ falls outside the forbidden interval $\left(\theta-\alpha_{x}, \theta+\alpha_{x}\right)$. The noise location $a$ replaces the noise mean in the forbidden-interval condition for infinite-variance noise. So adding noise in the form of squeezing noise, channel noise, and detector inefficiency noise can enhance the performance of the quantum communication system. Figure 1 shows a simulation instance of the if-part of Theorem 1 .

The theorem states that the mutual information $I(S, Y)$ between sender and receiver tends to zero as all noise parameters decrease to zero. The theorem assumes that the input and output signals are statistically dependent so that $I(S, Y)>0$ where $I(S, Y)=$ $\sum_{s, y} p_{S, Y}(s, y) \log \left(p_{S, Y}(s, y) /\left(p_{S}(s) p_{Y}(y)\right)\right)$ 15]. So the SR effect occurs because the mutual information $I(S, Y)$ must increase from zero as we add noise to the system: what goes down must go up. We state the parameters for the finite-variance case without parentheses and the parameters for the infinite-variance case with parentheses.

Theorem 1 Suppose the position quadrature $\nu_{x}$ of the channel noise has finite variance $\sigma_{\nu_{x}}^{2}$ and mean $\mu_{\nu_{x}}$ (dispersion $\gamma$ and location a). Suppose the input signal's position quadrature $\alpha_{x}$ is subthreshold: $\alpha_{x}<\theta$. Suppose there is some statistical dependence between input signal $S$ and output signal $Y$ so that the mutual information obeys $I(S, Y)>0$. Then the quantum communication system exhibits the nonmonotone SR effect if and only if the position quadrature of the noise mean (location) does not lie in the forbidden interval: $\mu_{\nu_{x}} \notin\left(\theta-\alpha_{x}, \theta+\alpha_{x}\right)$ $\left(a \notin\left(\theta-\alpha_{x}, \theta+\alpha_{x}\right)\right)$. The nonmonotone $S R$ effect is that $I(S, Y) \rightarrow 0$ as $\sigma_{\nu_{x}}^{2} \rightarrow 0(\gamma \rightarrow 0)$, as $r \rightarrow \infty$, and as $\eta \rightarrow 1$.

Proof. The finite-variance proof for sufficiency and necessity follows the respective proofs in [7] and [8] if we use $p_{N}(n)$ as the noise density. The infinite-variance proof follows the respective stable proofs in [7] and [8] if we use $p_{N}(n)$ as the noise density and if $\nu_{x}$ is an alpha-stable random variable. Only slight modifications of the proofs account for the homodyne efficiency $\eta$. See Appendix IA for the finite-variance proof and Appendix $\mathrm{IB}$ for the infinite-variance proof.

SR in Continuous-Variable Quantum Key Distribution - The SR effect occurs in the continuous-variable quantum key distribution (CVQKD) scenario from [12, 13]. This CVQKD model thresholds a continuous parameter to establish a secret key between Alice and Bob. We modify the form of the above forbidden-interval theorem to include the subtleties of the CVQKD model. The re- sulting theorem gives necessary and sufficient conditions for the SR effect in CVQKD.

The theorems have security implications for CVQKD with thresholding. Suppose that $I(A, B)$ is the mutual information between Alice and Bob and that $I(A, E)$ is the mutual information between Alice and an attacker Eve. The SR effect influences the privacy condition $I(A, B)>I(A, E)$ 21] because it affects $I(A, B)$.

We first present the model for CVQKD from [12, 13. without including the attacker Eve. Alice wants to send a random secret bit $S$ to Bob. Alice randomly sends one of four coherent states to Bob: $\{|\alpha\rangle,|i \alpha\rangle,|-\alpha\rangle,|-i \alpha\rangle\}$ where $\alpha \in \mathbb{R}^{+}$. Random bit $S=0$ if she sends $|-\alpha\rangle$ or $|-i \alpha\rangle$ and $S=1$ if she sends $|\alpha\rangle$ or $|i \alpha\rangle$. Bob randomly measures the state's position quadrature or momentum quadrature. Alice and Bob communicate classically after Alice sends a large quantity of quantum data to Bob. They divide the measurement results into "correct-basis" and "incorrect-basis." The data is correct-basis if Bob measures the position quadrature when Alice sends $\{|\alpha\rangle,|-\alpha\rangle\}$ or if Bob measures the momentum quadrature when Alice sends $\{|i \alpha\rangle,|-i \alpha\rangle\}$. The data is incorrect-basis if it is not correct-basis. Alice and Bob keep only correct-basis data. Let $x \in \mathbb{R}$ be the result of Bob's measurement. Bob sets a threshold $\theta$ and assigns a bit value $Y$ where $Y=1$ if $x \geq \theta, Y=0$ if $x \leq-\theta$, and $Y=\varepsilon$ otherwise. Symbol $\varepsilon$ represents an inconclusive result.

Our analysis below corresponds only to correct-basis data because this data is crucial for determining the resulting performance of the protocol. We present the analysis only for the position-quadrature basis case. The same analysis holds for the momentum-quadrature case.

We now present a Heisenberg-picture analysis of the above model and include strategies that the attacker Eve can employ. The first few steps begin in the same way as the basic protocol above with Eve controlling the noisy channel. Then $\hat{x} e^{-r}+(-1)^{S+1} \alpha_{x}+\nu_{x}$ is the positionquadrature observable for the state that Eve possesses. She performs an amplifier-beamsplitter attack [22] by first passing the state through a phase-insensitive linear amplifier with gain $G \geq 1[23]$. She then leaks a fraction $1-\eta_{E}$ of the state through a beamsplitter so that Bob receives the fraction $\eta_{E}$. The Heisenberg-picture observable that corresponds to Bob's state is

$$
\sqrt{\eta_{E} G} \hat{x}_{s}+\sqrt{\eta_{E}(G-1)} \hat{x}_{E_{1}}+\sqrt{1-\eta_{E}} \hat{x}_{E_{2}}
$$

where $\hat{x}_{s}=\hat{x} e^{-r}+(-1)^{S+1} \alpha+\nu_{x}$. Modes $\hat{x}_{E_{1}}$ and $\hat{x}_{E_{2}}$ are vacuum modes resulting from the amplifier and beamsplitter and correspond to zero-mean $1 / 2$-variance Gaussian random variables upon measurement. Bob then measures the above operator by non-ideal positionquadrature homodyne detection. It collapses to the random variable $N+\sqrt{\eta_{E} \eta_{B} G}(-1)^{S+1} \alpha$ where $N$ sums all 
noise terms

$$
\begin{array}{r}
N \equiv \sqrt{\eta_{E} \eta_{B} G}\left(X e^{-r}+\nu_{x}\right)+\sqrt{\eta_{E} \eta_{B}(G-1)} X_{E_{1}}+ \\
\sqrt{\eta_{B}\left(1-\eta_{E}\right)} X_{E_{2}}+\sqrt{1-\eta_{B}} X_{H},
\end{array}
$$

$\eta_{B}$ is the efficiency of Bob's homodyne detection, and $X_{H}$ is a zero-mean $1 / 2$-variance Gaussian random variable that arises from homodyne detection noise. The density $p_{N}(n)$ of random variable $N$ is

$$
p_{N}(n)=\left(p_{\mathcal{N}\left(0, \sigma^{2}\right)} * p_{\sqrt{\eta_{E} \eta_{B} G \nu_{x}}}\right)(n)
$$

where $p_{\mathcal{N}\left(0, \sigma^{2}\right)}$ is the density of a zero-mean Gaussian random variable with variance

$$
\left(\eta_{B}\left(\eta_{E} G e^{-2 r}+\eta_{E}(G-1)+\left(1-\eta_{E}\right)\right)+1-\eta_{B}\right) / 2
$$

and $p_{\sqrt{\eta_{E} \eta_{B} G} \nu_{x}}$ is the density of $\sqrt{\eta_{E} \eta_{B} G} \nu_{x}$. Bob decodes with a threshold $\theta$ and gets a random bit $Y$ where

$$
Y= \begin{cases}1: & N+\sqrt{\eta_{E} \eta_{B} G}(-1)^{S+1} \alpha \geq \theta \\ 0 & : N+\sqrt{\eta_{E} \eta_{B} G}(-1)^{S+1} \alpha \leq-\theta . \\ \varepsilon & : \text { else }\end{cases}
$$

Protagonists Alice and Bob and antagonist Eve all play a role in the SR effect in Alice and Bob's communication of a secret key. Alice can add Heisenberg noise in the form of squeezing. Eve can add channel, amplifier, and leakage noise in her attack. Bob can add photodetector inefficiency noise. The modified quantum forbiddeninterval theorem characterizes this interplay and gives a necessary and sufficient condition for the SR effect. Figure 2 shows a simulation instance of the if-part of the theorem.

Theorem 2 Suppose the channel noise position quadrature has finite variance $\sigma_{\nu_{x}}^{2}$ and mean $\mu_{\nu_{x}}$ (dispersion $\gamma$ and location a). Suppose the input signal's amplitude $\alpha$ is subthreshold: $\alpha<\theta$ and $-\alpha>-\theta$. Suppose there is some statistical dependence between input signal $S$ and output signal $Y$ so that the mutual information obeys $I(S, Y)>$ 0 . Then the quantum key distribution system exhibits the nonmonotone SR effect if and only if the position quadrature of the noise mean (location) does not lie in the forbidden interval: $\mu_{\nu_{x}} \notin(-\theta-\alpha,-\theta+\alpha) \cup(\theta-\alpha, \theta+\alpha)$ $(a \notin(-\theta-\alpha,-\theta+\alpha) \cup(\theta-\alpha, \theta+\alpha))$. The nonmonotone $S R$ effect is that $I(S, Y) \rightarrow 0$ as $\sigma_{\nu_{x}}^{2} \rightarrow 0(\gamma \rightarrow 0)$, as $r \rightarrow \infty$, as $G \rightarrow 1$, as $\eta_{E} \rightarrow 1$, and as $\eta_{B} \rightarrow 1$.

Proof. The proof method follows the proof of Theorem 1 using $p_{N}(n)$ in (8). The proof requires three cases rather than two because of the differences between CVQKD and the basic model. See Appendix IC for the finite-variance proof and Appendix ID for the infinite-variance proof.

Conclusion-Theorems 11 and 2 guarantee only that the nonmonotone SR effect occurs. They do not give the optimal combination of channel noise, squeezing, and photodetector inefficiency noise. Nor do they guarantee a large increase in mutual information. The theorems

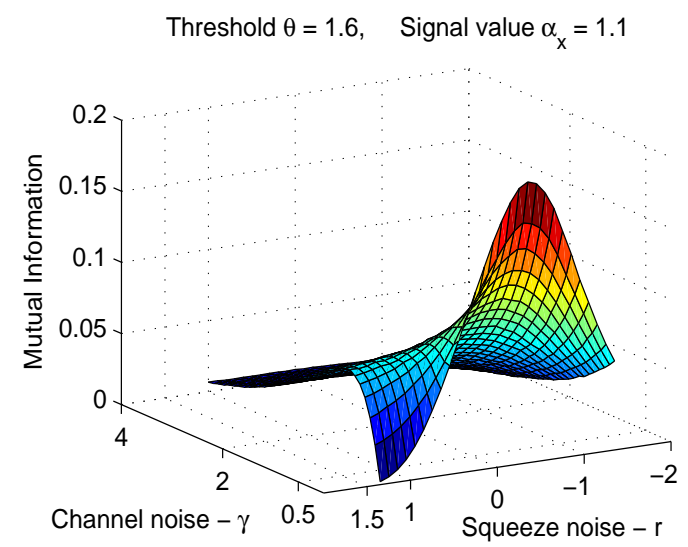

FIG. 2: (Color online) SR in continuous-variable quantum key distribution. Alice encodes coherent states with amplitude $A=1.1$ and Bob decodes with threshold $\theta=1.6$. The graph shows the smoothed mutual information as a function of the dispersion $\gamma$ of infinite-variance quantum Cauchy noise and squeezing strength $r$ for 100 simulation runs. We do not include amplifier, beamsplitter, or photodetector inefficiency noise. Each run generated 10,000 input-output signal pairs to estimate the mutual information. The SR effect occurs because the channel noise location $a=0$ and so $a$ lies outside the forbidden interval $(-2.7,-.5) \cup(.5,2.7)$.

also may not appear realistic because their proof requires infinite squeezing in the limit and thus requires infinite energy. But the theorems guarantee that the SR effect occurs for some finite squeezing. The simulations in both figures display the full nonmonotone SR signature for experimentally plausible squeezing values and for realistic channel noise levels.

Forbidden interval theorems may hold for more complex quantum systems. The quantum systems in this paper use noisy quantum processing to produce a mutual-information benefit between two classical variables. Other systems might use noise to enhance the fidelity of the coherent superposition of a quantum state. The performance measure would be the coherent information [24] because it corresponds operationally to the capacity of a quantum channel [25]. The coherent information also relates to the quantum channel capacity for sending private classical information [25]. This suggests further connections between SR and QKD and the potential for new learning algorithms that can locate any noise optima.

The authors thank Todd A. Brun, Igor Devetak, Jonathan P. Dowling, and Austin Lund for helpful discussions. MMW acknowledges support from NSF Grant CCF-0545845, the Hearne Institute for Theoretical Physics, Army Research Office, and Disruptive Technologies Office. 


\section{APPENDIX}

\section{A. Proof of Theorem 1 (Finite Variance)}

The proofs for sufficiency and necessity follow the respective proof methods in (7] and [8] if we use (5) as the noise density.

Let us calculate the four conditional probabilities $p_{Y \mid S}(0 \mid 0), p_{Y \mid S}(0 \mid 1), p_{Y \mid S}(1 \mid 0), p_{Y \mid S}(1 \mid 1)$.

$$
\begin{aligned}
& p_{Y \mid S}(0 \mid 0) \\
& =\operatorname{Pr}\left\{u\left(\sqrt{\eta}(-1)^{S+1} \alpha_{x}+N-\theta\right) \mid S=0\right\} \\
& =\operatorname{Pr}\left\{\sqrt{\eta}(-1)^{S+1} \alpha_{x}+N-\theta<0 \mid S=0\right\} \\
& =\operatorname{Pr}\left\{-\sqrt{\eta} \alpha_{x}+N<\theta\right\}=\operatorname{Pr}\left\{N<\theta+\sqrt{\eta} \alpha_{x}\right\} \\
& =\int_{-\infty}^{\theta+\sqrt{\eta} \alpha_{x}} p_{N}(n) d n
\end{aligned}
$$

The other conditional probabilities follow from similar calculations:

$$
\begin{aligned}
& p_{Y \mid S}(0 \mid 1)=\int_{-\infty}^{\theta-\sqrt{\eta} \alpha_{x}} p_{N}(n) d n \\
& p_{Y \mid S}(1 \mid 0)=\int_{\theta+\sqrt{\eta} \alpha_{x}}^{\infty} p_{N}(n) d n \\
& p_{Y \mid S}(1 \mid 1)=\int_{\theta-\sqrt{\eta} \alpha_{x}}^{\infty} p_{N}(n) d n
\end{aligned}
$$

Proof (Sufficiency). Assume that $0<p_{S}(s)<1$ to avoid triviality when $p_{S}(s)=0$ or $1 . \quad I(S, Y)=0$ if and only if $S$ and $Y$ are statistically independent [15]. We show that $S$ and $Y$ are asymptotically independent: $I(S, Y) \rightarrow 0$ as $\sigma_{\nu_{x}}^{2} \rightarrow 0$, as $r \rightarrow \infty$, and as $\eta \rightarrow 1$. The following definition holds for the proofs that follow:

$$
\sigma^{2} \equiv \eta\left(e^{-2 r} / 2+\sigma_{\nu_{x}}^{2}\right)+(1-\eta) / 2
$$

We need to show that $p_{Y \mid S}(y \mid s)=p_{Y}(y)$ as $\sigma_{\nu_{x}}^{2} \rightarrow 0$, as $r \rightarrow \infty$, and as $\eta \rightarrow 1$ for $s, y \in\{0,1\}$. Consider an algebraic manipulation using the law of total probability:

$$
\begin{aligned}
p_{Y}(y) & =\sum_{s} p_{Y \mid S}(y \mid s) p_{S}(s) \\
& =p_{Y \mid S}(y \mid 0) p_{S}(0)+p_{Y \mid S}(y \mid 1) p_{S}(1) \\
& =p_{Y \mid S}(y \mid 0) p_{S}(0)+p_{Y \mid S}(y \mid 1)\left(1-p_{S}(0)\right) \\
& =\left(p_{Y \mid S}(y \mid 0)-p_{Y \mid S}(y \mid 1)\right) p_{S}(0)+p_{Y \mid S}(y \mid 1)
\end{aligned}
$$

We can show by a similar method that

$$
p_{Y}(y)=\left(p_{Y \mid S}(y \mid 1)-p_{Y \mid S}(y \mid 0)\right) p_{S}(1)+p_{Y \mid S}(y \mid 0)
$$

So $p_{Y}(y) \rightarrow p_{Y \mid S}(y \mid 1)$ and $p_{Y}(y) \rightarrow p_{Y \mid S}(y \mid 0)$ as $p_{Y \mid S}(y \mid 1)-p_{Y \mid S}(y \mid 0) \rightarrow 0$. Consider the case where $y=0$.

$$
p_{Y \mid S}(0 \mid 0)-p_{Y \mid S}(0 \mid 1)=\int_{\theta-\sqrt{\eta} \alpha_{x}}^{\theta+\sqrt{\eta} \alpha_{x}} p_{N}(n) d n
$$

Consider the case where $y=1$.

$$
p_{Y \mid S}(1 \mid 0)-p_{Y \mid S}(1 \mid 1)=-\int_{\theta-\sqrt{\eta} \alpha_{x}}^{\theta+\sqrt{\eta} \alpha_{x}} p_{N}(n) d n
$$

So the result follows if

$$
\int_{\theta-\sqrt{\eta} \alpha_{x}}^{\theta+\sqrt{\eta} \alpha_{x}} p_{N}(n) d n \rightarrow 0
$$

as $\sigma_{\nu_{x}}^{2} \rightarrow 0$, as $r \rightarrow \infty$, and as $\eta \rightarrow 1$. Suppose the mean

$$
\mu_{\nu_{x}} \notin\left(\theta-\alpha_{x}, \theta+\alpha_{x}\right)
$$

by hypothesis. We ignore the zero-measure cases where $\mu_{\nu_{x}}=\theta-\alpha_{x}$ or $\mu_{\nu_{x}}=\theta+\alpha_{x}$.

Case 1: Suppose first that $\mu_{\nu_{x}}<\theta-\alpha_{x}$. So $\mu_{\nu_{x}}+\alpha_{x}<$ $\theta$ and thus

$$
\sqrt{\eta}\left(\mu_{\nu_{x}}+\alpha_{x}\right) \leq \mu_{\nu_{x}}+\alpha_{x}<\theta
$$

for any $\eta \in(0,1]$. Pick

$$
\epsilon=\frac{1}{2}\left(\theta-\sqrt{\eta} \alpha_{x}-\sqrt{\eta} \mu_{\nu_{x}}\right)>0 .
$$

So $\theta-\sqrt{\eta} \alpha_{x}-\epsilon=\sqrt{\eta} \mu_{x}+\epsilon$. Then

$$
\begin{aligned}
& \int_{\theta-\sqrt{\eta} \alpha_{x}}^{\theta+\sqrt{\eta} \alpha_{x}} p_{N}(n) d n \\
& \leq \int_{\theta-\sqrt{\eta} \alpha_{x}}^{\infty} p_{N}(n) d n \\
& \leq \int_{\theta-\sqrt{\eta} \alpha_{x}-\epsilon}^{\infty} p_{N}(n) d n \\
& \leq \int_{\sqrt{\eta} \mu_{\nu_{x}}+\epsilon}^{\infty} p_{N}(n) d n \\
& =\operatorname{Pr}\left\{N \geq \sqrt{\eta} \mu_{\nu_{x}}+\epsilon\right\} \\
& =\operatorname{Pr}\{N \geq \mu+\epsilon\} \\
& =\operatorname{Pr}\{N-\mu \geq \epsilon\} \\
& \leq \operatorname{Pr}\{|N-\mu| \geq \epsilon\} \\
& \leq \frac{\sigma^{2}}{\epsilon^{2}}
\end{aligned}
$$

So the result follows when $\mu_{\nu_{x}}<\theta-\alpha_{x}$ because $p_{Y \mid S}(0 \mid 0)-p_{Y \mid S}(0 \mid 1) \rightarrow 0$ as $\sigma_{\nu_{x}}^{2} \rightarrow 0$, as $r \rightarrow \infty$, and as $\eta \rightarrow 1$.

Case 2: Suppose next that $\mu_{\nu_{x}}>\theta+\alpha_{x}$ so that $\mu_{\nu_{x}}-\alpha_{x}>\theta>0$. Choose $\sqrt{\eta}$ large enough so that

$$
\sqrt{\eta}>\theta /\left(\mu_{\nu_{x}}-\alpha_{x}\right)
$$

So $\sqrt{\eta}\left(\mu_{\nu_{x}}-\alpha_{x}\right)>\theta$. Pick

$$
\epsilon=\frac{1}{2}\left(\sqrt{\eta} \mu_{\nu_{x}}-\theta-\sqrt{\eta} \alpha_{x}\right)>0
$$


So $\theta+\sqrt{\eta} \alpha_{x}+\epsilon=\sqrt{\eta} \mu_{\nu_{x}}-\epsilon$. Then

$$
\begin{aligned}
& \int_{\theta-\sqrt{\eta} \alpha_{x}}^{\theta+\sqrt{\eta} \alpha_{x}} p_{N}(n) d n \\
& \leq \int_{-\infty}^{\theta+\sqrt{\eta} \alpha_{x}} p_{N}(n) d n \\
& \leq \int_{-\infty}^{\theta+\sqrt{\eta} \alpha_{x}+\epsilon} p_{N}(n) d n \\
& \leq \int_{-\infty}^{\sqrt{\eta} \mu_{\nu_{x}}-\epsilon} p_{N}(n) d n \\
& =\operatorname{Pr}\left\{N \leq \sqrt{\eta} \mu_{\nu_{x}}-\epsilon\right\} \\
& =\operatorname{Pr}\{N \leq \mu-\epsilon\} \\
& =\operatorname{Pr}\{N-\mu \leq-\epsilon\} \\
& \leq \operatorname{Pr}\{|N-\mu| \geq \epsilon\} \\
& \leq \frac{\sigma^{2}}{\epsilon^{2}}
\end{aligned}
$$

So $p_{Y \mid S}(0 \mid 0)-p_{Y \mid S}(0 \mid 1) \rightarrow 0$ as $\sigma_{\nu_{x}}^{2} \rightarrow 0$, as $r \rightarrow \infty$, and as $\eta \rightarrow 1$ when $\mu_{\nu_{x}}>\theta+\alpha_{x}$. Thus

$$
\mu_{\nu_{x}} \notin\left(\theta-\alpha_{x}, \theta+\alpha_{x}\right)
$$

is a sufficient condition for the nonmonotone SR effect to occur.

Proof (Necessity). The system does not exhibit the nonmonotone SR effect if $\mu_{\nu_{x}} \in\left(\theta-\alpha_{x}, \theta+\alpha_{x}\right)$ in the sense that $I(S, Y)$ is maximum as $\sigma_{\nu_{x}}^{2} \rightarrow 0$, as $r \rightarrow \infty$, and as $\eta \rightarrow 1$. $I(S, Y) \rightarrow H(Y)=H(S)$ as $\sigma_{\nu_{x}}^{2} \rightarrow 0$, as $r \rightarrow \infty$, and as $\eta \rightarrow 1$. Assume that $0<p_{S}(s)<$ 1 to avoid triviality when $p_{S}(s)=0$ or 1 . We show that $H(Y) \rightarrow H(S)$ and $H(Y \mid S) \rightarrow 0$ as $\sigma_{\nu_{x}}^{2} \rightarrow 0$, as $r \rightarrow \infty$, and as $\eta \rightarrow 1$. It is maximum in this limit because $I(S, Y)=H(Y)-H(Y \mid S)$ and $I(S, Y) \leq H(S)$ by the data processing inequality for a Markov chain [15]. Consider the conditional entropy $H(Y \mid S)$ :

$$
\begin{aligned}
& H(Y \mid S) \\
& =-\sum_{s, y} p_{Y, S}(y, s) \log _{2} p_{Y \mid S}(y \mid s) \\
& =-\sum_{s} p_{S}(s) \sum_{y} p_{Y \mid S}(y \mid s) \log _{2} p_{Y \mid S}(y \mid s)
\end{aligned}
$$

Suppose for now that $p_{Y \mid S}(y \mid s) \rightarrow 1$ or 0 for all $s, y \in$ $\{0,1\}$ as $\sigma_{\nu_{x}}^{2} \rightarrow 0$, as $r \rightarrow \infty$, and as $\eta \rightarrow 1$. Then $H(Y \mid S) \rightarrow 0$ by inspecting (17) and applying $1 \log _{2} 1=0$ and $0 \log _{2} 0=0$ by L'Hôspital's rule. So we aim to prove that each of the conditional probabilities vanish or approach 1 in the above limit if $\mu_{\nu_{x}} \in\left(\theta-\alpha_{x}, \theta+\alpha_{x}\right)$. Consider first $p_{Y \mid S}(0 \mid 0)$. Pick any $\mu_{\nu_{x}} \in\left(\theta-\alpha_{x}, \theta+\alpha_{x}\right)$. Then $\theta+\alpha_{x}-\mu_{\nu_{x}}>0$ and $\theta>\mu_{\nu_{x}}-\alpha_{x}$. Then $\theta>\sqrt{\eta}\left(\mu_{\nu_{x}}-\alpha_{x}\right)$ for any $\eta \in(0,1]$. Pick $\epsilon=$ $\frac{1}{2}\left(\theta+\sqrt{\eta} \alpha_{x}-\sqrt{\eta} \mu_{\nu_{x}}\right)>0$ so that $\theta+\sqrt{\eta} \alpha_{x}-\epsilon=$ $\sqrt{\eta} \mu_{\nu_{x}}+\epsilon$

$$
\begin{aligned}
p_{Y \mid S}(0 \mid 0) & \\
= & \int_{-\infty}^{\theta+\sqrt{\eta} \alpha_{x}} p_{N}(n) d n \\
\geq & \int_{-\infty}^{\theta+\sqrt{\eta} \alpha_{x}-\epsilon} p_{N}(n) d n \\
= & \int_{-\infty}^{\sqrt{\eta} \mu_{\nu_{x}}+\epsilon} p_{N}(n) d n \\
= & 1-\int_{\sqrt{\eta} \mu_{\nu_{x}}+\epsilon}^{\infty} p_{N}(n) d n \\
= & 1-\operatorname{Pr}\left\{N \geq \sqrt{\eta} \mu_{\nu_{x}}+\epsilon\right\} \\
= & 1-\operatorname{Pr}\{N \geq \mu+\epsilon\} \\
= & 1-\operatorname{Pr}\{N-\mu \geq \epsilon\} \\
\geq & 1-\operatorname{Pr}\{|N-\mu| \geq \epsilon\} \\
\geq & 1-\frac{\sigma^{2}}{\epsilon^{2}} \\
\rightarrow & 1
\end{aligned}
$$

as $\sigma_{\nu_{x}}^{2} \rightarrow 0$,as $r \rightarrow \infty$,and as $\eta \rightarrow 1$. We prove the result similarly for $p_{Y \mid S}(1 \mid 1)$. Pick any $\mu_{\nu_{x}} \in\left(\theta-\alpha_{x}, \theta+\alpha_{x}\right)$. Then $\mu_{\nu_{x}}>\theta-\alpha_{x}$ and $\mu_{\nu_{x}}+\alpha_{x}>\theta>0$. Suppose that $\eta$ is large enough so that $\sqrt{\eta}>\theta /\left(\mu_{\nu_{x}}+\alpha_{x}\right)$. Then $\sqrt{\eta}\left(\mu_{\nu_{x}}+\alpha_{x}\right)>\theta$ and $\sqrt{\eta} \mu_{\nu_{x}}+\sqrt{\eta} \alpha_{x}-\theta>0$. Pick $\epsilon=\frac{1}{2}\left(\sqrt{\eta} \mu_{\nu_{x}}+\sqrt{\eta} \alpha_{x}-\theta\right)>0$ so that $\theta-\sqrt{\eta} \alpha_{x}+\epsilon=$ $\sqrt{\eta} \mu_{\nu_{x}}-\epsilon$.

$$
\begin{aligned}
p_{Y \mid S}(1 \mid 1) \\
=\int_{\theta-\sqrt{\eta} \alpha_{x}}^{\infty} p_{N}(n) d n \\
\geq \int_{\theta-\sqrt{\eta} \alpha_{x}+\epsilon}^{\infty} p_{N}(n) d n \\
=\int_{\sqrt{\eta} \mu_{\nu_{x}}-\epsilon}^{\infty} p_{N}(n) d n \\
=1-\int_{-\infty}^{\sqrt{\eta} \mu_{\nu_{x}}-\epsilon} p_{N}(n) d n \\
=1-\operatorname{Pr}\left\{N \leq \sqrt{\eta} \mu_{\nu_{x}}-\epsilon\right\} \\
=1-\operatorname{Pr}\{N \leq \mu-\epsilon\} \\
=1-\operatorname{Pr}\{N-\mu \leq-\epsilon\} \\
\geq 1-\operatorname{Pr}\{|N-\mu| \geq \epsilon\} \\
\geq 1-\frac{\sigma^{2}}{\epsilon^{2}} \\
\rightarrow 1
\end{aligned}
$$

as $\sigma_{\nu_{x}}^{2} \rightarrow 0$,as $r \rightarrow \infty$, and as $\eta \rightarrow 1$. So $p_{Y \mid S}(0 \mid 0) \rightarrow$ $1, p_{Y \mid S}(1 \mid 1) \rightarrow 1, p_{Y \mid S}(1 \mid 0) \rightarrow 0$, and $p_{Y \mid S}(0 \mid 1) \rightarrow 0$ as $\sigma_{\nu_{x}}^{2} \rightarrow 0$, as $r \rightarrow \infty$, and as $\eta \rightarrow 1$ and the system does not display the nonmonotone SR effect. 


\section{B. Proof of Theorem 1 (Infinite Variance)}

The proofs for sufficiency and necessity follow the respective stable proof methods in [7] and [8] if we use (5) as the noise density and if $\nu_{x}$ is an alpha-stable random variable.

The characteristic function $\varphi_{\nu_{x}}(\omega)$ of an alpha-stable noise source with density $p_{\nu_{x}}(n)$ is the following:

$$
\begin{aligned}
& \varphi_{\nu_{x}}(\omega)= \\
& \quad \exp \left\{i a \omega-\gamma|\omega|^{\alpha}\left(1+i \beta \operatorname{sign}(\omega) \tan \left(\frac{\alpha \pi}{2}\right)\right)\right\}
\end{aligned}
$$

where $\alpha$ is the characteristic exponent and $\beta$ is a skewness parameter. The characteristic function of $p_{N}(n)$ is as follows

$$
\begin{aligned}
& \varphi_{N}(\omega)
\end{aligned}
$$

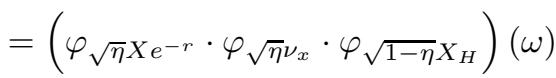

$$
\begin{aligned}
& =\exp \left\{-\frac{\eta e^{-2 r} \omega^{2}}{4}\right\} \varphi_{\nu_{x}}(\sqrt{\eta} \omega) \exp \left\{-\frac{(1-\eta) \omega^{2}}{4}\right\} \\
& =\varphi_{\nu_{x}}(\sqrt{\eta} \omega) \exp \left\{-\frac{\left(\eta e^{-2 r}+1-\eta\right) \omega^{2}}{4}\right\}
\end{aligned}
$$

from (5) and the convolution theorem.

Proof (Sufficiency). Take the limit of the characteristic function $\varphi_{N}(\omega)$ as $\gamma \rightarrow 0$, as squeezing parameter $r \rightarrow \infty$, and as homodyne efficiency $\eta \rightarrow 1$ to obtain the following characteristic function.

$$
\lim _{r \rightarrow \infty, \gamma \rightarrow 0, \eta \rightarrow 1} \varphi_{N}(\omega)=\exp \{i a \omega\}
$$

The probability density $p_{N}(n)$ then approaches a translated delta function

$$
\lim _{r \rightarrow \infty, \gamma \rightarrow 0, \eta \rightarrow 1} p_{N}(n)=\delta(n-a)
$$

The conditional probability difference obeys:

$$
\begin{aligned}
p_{Y \mid S}(0 \mid 0)-p_{Y \mid S}(0 \mid 1) & =\int_{\theta-\sqrt{\eta} \alpha_{x}}^{\theta+\sqrt{\eta} \alpha_{x}} p_{N}(n) d n \\
& \leq \int_{\theta-\alpha_{x}}^{\theta+\alpha_{x}} p_{N}(n) d n
\end{aligned}
$$

Pick $a \notin\left(\theta-\alpha_{x}, \theta+\alpha_{x}\right)$. Consider the following limit:

$$
\begin{aligned}
& \lim _{r \rightarrow \infty, \gamma \rightarrow 0, \eta \rightarrow 1} p_{Y \mid S}(0 \mid 0)-p_{Y \mid S}(0 \mid 1) \\
& \leq \lim _{r \rightarrow \infty, \gamma \rightarrow 0, \eta \rightarrow 1} \int_{\theta-\alpha_{x}}^{\theta+\alpha_{x}} p_{N}(n) d n \\
& =\int_{\theta-\alpha_{x}}^{\theta+\alpha_{x}} \delta(n-a) d n=0
\end{aligned}
$$

Proof (Necessity). Choose $a \in\left(\theta-\alpha_{x}, \theta+\alpha_{x}\right)$. Then

$$
\begin{aligned}
p_{Y \mid S}(0 \mid 0) & =\int_{-\infty}^{\theta+\sqrt{\eta} \alpha_{x}} p_{N}(n) d n \\
& =\int_{-\infty}^{\theta} p_{N}\left(n+\sqrt{\eta} \alpha_{x}\right) d n \\
& \rightarrow \int_{-\infty}^{\theta} \delta\left(n-a+\alpha_{x}\right) d n \\
& =\int_{-\infty}^{\theta+\alpha_{x}} \delta(n-a) d n=1 \\
\text { as } \gamma & \rightarrow 0, \text { as } r \rightarrow \infty, \text { and as } \eta \rightarrow 1 \\
p_{Y \mid S}(1 \mid 1) & =\int_{\theta-\sqrt{\eta} \alpha_{x}}^{\infty} p_{N}(n) d n \\
& =\int_{\theta}^{\infty} p_{N}\left(n-\sqrt{\eta} \alpha_{x}\right) d n \\
& \rightarrow \int_{\theta}^{\infty} \delta\left(n-a_{x}-\alpha_{x}\right) d n \\
& =\int_{\theta-\alpha_{x}}^{\infty} \delta\left(n-a_{x}\right) d n=1
\end{aligned}
$$

as $\gamma \rightarrow 0$, as $r \rightarrow \infty$, and as $\eta \rightarrow 1$

because $a \notin\left(\theta-\alpha_{x}, \theta+\alpha_{x}\right)$. 


\section{Proof of Theorem 2 (Finite Variance)}

The mean $\mu$ and variance $\sigma^{2}$ of noise random variable $N$ are $\mu=\sqrt{\eta_{E} \eta_{B} G} \mu_{\nu_{x}}$ and

$$
\begin{aligned}
& \sigma^{2}=\eta_{E} \eta_{B} G \sigma_{\nu_{x}}^{2}+ \\
& \quad\left(\eta_{B}\left(\begin{array}{c}
\eta_{E} G e^{-2 r}+\eta_{E}(G-1) \\
+\left(1-\eta_{E}\right)
\end{array}\right)+1-\eta_{B}\right) / 2
\end{aligned}
$$

We compute the six conditional probabilities: $p_{Y \mid S}(0 \mid 0)$, $p_{Y \mid S}(0 \mid 1), p_{Y \mid S}(1 \mid 0), p_{Y \mid S}(1 \mid 1), p_{Y \mid S}(\varepsilon \mid 0)$, and $p_{Y \mid S}(\varepsilon \mid 1)$.

$$
\begin{aligned}
& p_{Y \mid S}(0 \mid 0) \\
& =\operatorname{Pr}\left\{N+\sqrt{\eta_{E} \eta_{B} G}(-1)^{S+1} \alpha \leq-\theta \mid S=0\right\} \\
& =\operatorname{Pr}\left\{-\sqrt{\eta_{E} \eta_{B} G} \alpha+N \leq-\theta\right\} \\
& =\operatorname{Pr}\left\{N<-\theta+\sqrt{\eta_{E} \eta_{B} G} \alpha\right\} \\
& =\int_{-\infty}^{-\theta+\sqrt{\eta_{E} \eta_{B} G} \alpha} p_{N}(n) d n
\end{aligned}
$$

The other conditional probabilities follow from similar reasoning:

$$
\begin{aligned}
p_{Y \mid S}(0 \mid 1) & =\int_{-\infty}^{-\theta-\sqrt{\eta_{E} \eta_{B} G} \alpha} p_{N}(n) d n \\
p_{Y \mid S}(1 \mid 0) & =\int_{\theta+\sqrt{\eta_{E} \eta_{B} G} \alpha}^{\infty} p_{N}(n) d n \\
p_{Y \mid S}(1 \mid 1) & =\int_{\theta-\sqrt{\eta_{E} \eta_{B} G} \alpha}^{\infty} p_{N}(n) d n \\
p_{Y \mid S}(\varepsilon \mid 0) & =1-p_{Y \mid S}(0 \mid 0)-p_{Y \mid S}(1 \mid 0) \\
& =\int_{-\theta+\sqrt{\eta_{E} \eta_{B} G} \alpha}^{\theta+\sqrt{\eta_{E} \eta_{B} G} \alpha} p_{N}(n) d n \\
p_{Y \mid S}(\varepsilon \mid 1) & =1-p_{Y \mid S}(0 \mid 1)-p_{Y \mid S}(1 \mid 1) \\
& =\int_{-\theta-\sqrt{\eta_{E} \eta_{B} G} \alpha}^{\theta-\sqrt{\eta_{E} \eta_{B} G} \alpha} p_{N}(n) d n
\end{aligned}
$$

Proof (Sufficiency). We follow the proof method of Theorem [1] with some modifications. Note that the conditions $\eta_{E}, \eta_{B} \leq 1$ and $G \geq 1$ constrain the way in which we take both of their limits to one. We give the constraint on the values that the root of their product $\sqrt{\eta_{E} \eta_{B} G}$ may take for any given value of the noise mean $\mu_{\nu_{x}}$. Assume that these constraints are implicit when considering the limit in the proofs that follow.

Assume that $0<p_{S}(s)<1$ to avoid triviality when $p_{S}(s)=0$ or $1 . I(S, Y)=0$ if and only if $S$ and $Y$ are statistically independent [15]. We show that $S$ and $Y$ are asymptotically independent: $I(S, Y) \rightarrow 0$ as $\sigma_{\nu_{x}}^{2} \rightarrow 0$, as $r \rightarrow \infty$, as $\eta \rightarrow 1$, and as $G \rightarrow 1$. We need to show that
$p_{Y \mid S}(y \mid s) \rightarrow p_{Y}(y)$ as $\sigma_{\nu_{x}}^{2} \rightarrow 0$, as $r \rightarrow \infty$, as $\eta \rightarrow 1$, and as $G \rightarrow 1$ for $s, y \in\{0,1\}$. We do not consider $p_{Y \mid S}(y \mid \varepsilon)$ because the probability $p_{S}(\varepsilon)$ is zero and so the probability $p_{Y}(\varepsilon)$ is also zero. Consider the expansion in (15) using the law of total probability. The expansion is the same even when including symbol $\varepsilon$ because $\varepsilon$ has zero probability: $p_{S}(\varepsilon)=0$. So $p_{Y}(y) \rightarrow p_{Y \mid S}(y \mid 1)$ and $p_{Y}(y) \rightarrow p_{Y \mid S}(y \mid 0)$ as $p_{Y \mid S}(0 \mid 0)-p_{Y \mid S}(0 \mid 1) \rightarrow 0$ and $p_{Y \mid S}(1 \mid 1)-p_{Y \mid S}(1 \mid 0) \rightarrow 0$. Consider the case where $y=0$.

$$
p_{Y \mid S}(0 \mid 0)-p_{Y \mid S}(0 \mid 1)=\int_{-\theta-\sqrt{\eta_{E} \eta_{B} G} \alpha}^{-\theta+\sqrt{\eta_{E} \eta_{B} G} \alpha} p_{N}(n) d n
$$

Consider the case where $y=1$.

$$
p_{Y \mid S}(1 \mid 1)-p_{Y \mid S}(1 \mid 0)=\int_{\theta-\sqrt{\eta_{E} \eta_{B} G} \alpha}^{\theta+\sqrt{\eta_{E} \eta_{B} G} \alpha} p_{N}(n) d n
$$

So the result follows if both of the above conditional probability differences vanish as $\sigma_{\nu_{x}}^{2} \rightarrow 0$, as $r \rightarrow \infty$, as $\eta_{E}, \eta_{B} \rightarrow 1$, and as $G \rightarrow 1$. Suppose the mean $\mu_{\nu_{x}} \notin(-\theta-\alpha,-\theta+\alpha) \cup(\theta-\alpha, \theta+\alpha)$ by hypothesis. We ignore the zero-measure cases where $\mu_{\nu_{x}}=\theta-\alpha$, $\mu_{\nu_{x}}=\theta+\alpha, \mu_{\nu_{x}}=-\theta+\alpha$, or $\mu_{\nu_{x}}=-\theta-\alpha$.

Case 1: Suppose first that $\mu_{\nu_{x}}<-\theta-\alpha$. So $\mu_{\nu_{x}}+\alpha<-\theta$ and thus $\sqrt{\eta_{E} \eta_{B} G}\left(\mu_{\nu_{x}}+\alpha\right) \leq \mu_{\nu_{x}}+\alpha<-\theta$ whenever $\sqrt{\eta_{E} \eta_{B} G}>-\theta /\left(\mu_{\nu_{x}}+\alpha\right)=\theta /\left|\mu_{\nu_{x}}+\alpha\right|$. Pick $\epsilon=\frac{1}{2}\left(-\theta-\sqrt{\eta_{E} \eta_{B} G} \alpha-\sqrt{\eta_{E} \eta_{B} G} \mu_{\nu_{x}}\right)>0$. So $-\theta-\sqrt{\eta_{E} \eta_{B} G} \alpha-\epsilon=\sqrt{\eta_{E} \eta_{B} G} \mu_{\nu_{x}}+\epsilon$. Then

$$
\begin{aligned}
& \int_{-\theta-\sqrt{\eta_{E} \eta_{B} G} \alpha}^{-\theta+\sqrt{\eta_{E} \eta_{B} G} \alpha} p_{N}(n) d n \\
& \leq \int_{-\theta-\sqrt{\eta_{E} \eta_{B} G} \alpha}^{\infty} p_{N}(n) d n \\
& \leq \int_{-\theta-\sqrt{\eta_{E} \eta_{B} G} \alpha-\epsilon}^{\infty} p_{N}(n) d n \\
& \leq \int_{\sqrt{\eta_{E} \eta_{B} G} \mu_{\nu_{x}}+\epsilon}^{\infty} p_{N}(n) d n \\
& =\operatorname{Pr}\left\{N \geq \sqrt{\eta_{E} \eta_{B} G} \mu_{\nu_{x}}+\epsilon\right\} \\
& =\operatorname{Pr}\{N \geq \mu+\epsilon\} \\
& =\operatorname{Pr}\{N-\mu \geq \epsilon\} \\
& \leq \operatorname{Pr}\{|N-\mu| \geq \epsilon\} \\
& \leq \frac{\sigma^{2}}{\epsilon^{2}}
\end{aligned}
$$

So the conditional probability difference in (44) vanishes as $\sigma_{\nu_{x}}^{2} \rightarrow 0$, as $r \rightarrow \infty$, as $\eta_{E}, \eta_{B} \rightarrow 1$, and as $G \rightarrow 1$ when $\mu_{\nu_{x}}<-\theta-\alpha$. We now prove that the conditional probability difference in (45) vanishes when $\mu_{\nu_{x}}<-\theta-\alpha$. It follows that $\mu_{\nu_{x}}<\theta-\alpha$ if $\mu_{\nu_{x}}<$ $-\theta-\alpha$. So $\mu_{\nu_{x}}+\alpha<\theta$ and thus $\sqrt{\eta_{E} \eta_{B} G}\left(\mu_{\nu_{x}}+\alpha\right) \leq$ $\mu_{\nu_{x}}+\alpha<\theta$ for any $\sqrt{\eta_{E} \eta_{B} G} \geq 0$ because $\mu_{\nu_{x}}+\alpha<0$. 
Pick $\epsilon=\frac{1}{2}\left(\theta-\sqrt{\eta_{E} \eta_{B} G} \alpha-\sqrt{\eta_{E} \eta_{B} G} \mu_{\nu_{x}}\right)>0$. So $\theta-\sqrt{\eta_{E} \eta_{B} G} \alpha-\epsilon=\sqrt{\eta_{E} \eta_{B} G} \mu_{\nu_{x}}+\epsilon$. Then

$$
\begin{aligned}
& \int_{\theta-\sqrt{\eta_{E} \eta_{B} G \alpha}}^{\theta+\sqrt{\eta_{E} \eta_{B} G} \alpha} p_{N}(n) d n \\
& \leq \int_{\theta-\sqrt{\eta_{E} \eta_{B} G} \alpha}^{\infty} p_{N}(n) d n \\
& \leq \int_{\theta-\sqrt{\eta_{E} \eta_{B} G} \alpha-\epsilon}^{\infty} p_{N}(n) d n \\
& \leq \int_{\sqrt{\eta_{E} \eta_{B} G} \mu_{\nu_{x}}+\epsilon}^{\infty} p_{N}(n) d n \\
& =\operatorname{Pr}\left\{N \geq \sqrt{\eta_{E} \eta_{B} G} \mu_{\nu_{x}}+\epsilon\right\} \\
& =\operatorname{Pr}\{N \geq \mu+\epsilon\} \\
& =\operatorname{Pr}\{N-\mu \geq \epsilon\} \\
& \leq \operatorname{Pr}\{|N-\mu| \geq \epsilon\} \\
& \leq \frac{\sigma^{2}}{\epsilon^{2}}
\end{aligned}
$$

So the conditional probability difference in (45) vanishes as $\sigma_{\nu_{x}}^{2} \rightarrow 0$, as $r \rightarrow \infty$, as $\eta_{E}, \eta_{B} \rightarrow 1$, and as $G \rightarrow 1$ when $\mu_{\nu_{x}}<-\theta-\alpha$ and with constraint $\sqrt{\eta_{E} \eta_{B} G}>\theta /\left|\mu_{\nu_{x}}+\alpha\right|$.

Case 2: Suppose next that $-\theta+\alpha<\mu_{\nu_{x}}<\theta-\alpha$. We first prove that the conditional probability difference in (44) vanishes as $\sigma_{\nu_{x}}^{2} \rightarrow 0$, as $r \rightarrow \infty$, as $\eta_{E}, \eta_{B} \rightarrow 1$, and as $G \rightarrow 1$. So $\mu_{\nu_{x}}-\alpha>-\theta$ if $-\theta+\alpha<\mu_{\nu_{x}}<\theta-\alpha$. Thus $\sqrt{\eta_{E} \eta_{B} G}\left(\mu_{\nu_{x}}-\alpha\right) \geq$ $\mu_{\nu_{x}}-\alpha>-\theta$ whenever $\sqrt{\eta_{E} \eta_{B} G}<\theta /\left|\mu_{\nu_{x}}-\alpha\right|$. Pick $\epsilon=\frac{1}{2}\left(\theta-\sqrt{\eta_{E} \eta_{B} G} \alpha+\sqrt{\eta_{E} \eta_{B} G} \mu_{\nu_{x}}\right)>0$. So $-\theta+\sqrt{\eta_{E} \eta_{B} G} \alpha+\epsilon=\sqrt{\eta_{E} \eta_{B} G} \mu_{\nu_{x}}-\epsilon$. Then

$$
\begin{aligned}
& \int_{-\theta-\sqrt{\eta_{E} \eta_{B} G} \alpha}^{-\theta+\sqrt{\eta_{E} \eta_{B} G} \alpha} p_{N}(n) d n \\
& \leq \int_{-\infty}^{-\theta+\sqrt{\eta_{E} \eta_{B} G} \alpha} p_{N}(n) d n \\
& \leq \int_{-\infty}^{-\theta+\sqrt{\eta_{E} \eta_{B} G} \alpha+\epsilon} p_{N}(n) d n \\
& \leq \int_{-\infty}^{\sqrt{\eta_{E} \eta_{B} G} \mu_{\nu_{x}}-\epsilon} p_{N}(n) d n \\
& =\operatorname{Pr}\left\{N \leq \sqrt{\eta_{E} \eta_{B} G} \mu_{\nu_{x}}-\epsilon\right\} \\
& =\operatorname{Pr}\{N \leq \mu-\epsilon\} \\
& =\operatorname{Pr}\{N-\mu \leq-\epsilon\} \\
& \leq \operatorname{Pr}\{|N-\mu| \geq \epsilon\} \\
& \leq \frac{\sigma^{2}}{\epsilon^{2}}
\end{aligned}
$$

So the conditional probability difference in (44) vanishes as $\sigma_{\nu_{x}}^{2} \rightarrow 0$, as $r \rightarrow \infty$, as $\eta_{E}, \eta_{B} \rightarrow 1$, and as $G \rightarrow 1$ when $-\theta+\alpha<\mu_{\nu_{x}}<\theta-\alpha$. We now prove that the conditional probability difference in (45) vanishes as $\sigma_{\nu_{x}}^{2} \rightarrow 0$, as $r \rightarrow \infty$, as $\eta_{E}, \eta_{B} \rightarrow 1$, and as $G \rightarrow 1$. So $\mu_{\nu_{x}}+\alpha<\theta$ if $-\theta+\alpha<\mu_{\nu_{x}}<\theta-\alpha$. Thus $\sqrt{\eta_{E} \eta_{B} G}\left(\mu_{\nu_{x}}+\alpha\right) \leq$ $\mu_{\nu_{x}}+\alpha<\theta$ whenever $\sqrt{\eta_{E} \eta_{B} G}<\theta /\left|\mu_{\nu_{x}}+\alpha\right|$. Pick $\epsilon=\frac{1}{2}\left(\theta-\sqrt{\eta_{E} \eta_{B} G} \alpha-\sqrt{\eta_{E} \eta_{B} G} \mu_{\nu_{x}}\right)>0$. So $\theta-$ $\sqrt{\eta_{E} \eta_{B} G} \alpha-\epsilon=\sqrt{\eta_{E} \eta_{B} G} \mu_{\nu_{x}}+\epsilon$.

$$
\begin{aligned}
& \int_{\theta-\sqrt{\eta_{E} \eta_{B} G \alpha}}^{\theta+\sqrt{\eta_{E} \eta_{B} G} \alpha} p_{N}(n) d n \\
& \leq \int_{\theta-\sqrt{\eta_{E} \eta_{B} G \alpha}}^{\infty} p_{N}(n) d n \\
& \leq \int_{\theta-\sqrt{\eta_{E} \eta_{B} G} \alpha-\epsilon}^{\infty} p_{N}(n) d n \\
& \leq \int_{\sqrt{\eta_{E} \eta_{B} G} \mu_{\nu_{x}}+\epsilon}^{\infty} p_{N}(n) d n \\
& =\operatorname{Pr}\left\{N \geq \sqrt{\eta_{E} \eta_{B} G} \mu_{\nu_{x}}+\epsilon\right\} \\
& =\operatorname{Pr}\{N \geq \mu+\epsilon\} \\
& =\operatorname{Pr}\{N-\mu \geq \epsilon\} \\
& \leq \operatorname{Pr}\{|N-\mu| \geq \epsilon\} \\
& \leq \frac{\sigma^{2}}{\epsilon^{2}}
\end{aligned}
$$

So the conditional probability difference in (45) vanishes as $\sigma_{\nu_{x}}^{2} \rightarrow 0$, as $r \rightarrow \infty$, as $\eta_{E}, \eta_{B} \rightarrow 1$, and as $G \rightarrow 1$ when $-\theta+\alpha<\mu_{\nu_{x}}<\theta-\alpha$ and with the constraint $\sqrt{\eta_{E} \eta_{B} G} \leq \min \left(\theta /\left|\mu_{\nu_{x}}+\alpha\right|, \theta /\left|\mu_{\nu_{x}}-\alpha\right|\right)$.

Case 3: Suppose next that $\mu_{\nu_{x}}>\theta+\alpha$ so that $\mu_{\nu_{x}}-\alpha>\theta>0$. We first prove that the conditional probability difference in (44) vanishes as $\sigma_{\nu_{x}}^{2} \rightarrow 0$, as $r \rightarrow \infty$, as $\eta_{E}, \eta_{B} \rightarrow 1$, and as $G \rightarrow 1$. So $\mu_{\nu_{x}}>-\theta+\alpha$ if $\mu_{\nu_{x}}>\theta+\alpha$. Thus $\mu_{\nu_{x}}>-\theta+\alpha$ and $\mu_{\nu_{x}}-\alpha>-\theta$ and $\sqrt{\eta_{E} \eta_{B} G}\left(\mu_{\nu_{x}}-\alpha\right)>-\theta$ for any $\sqrt{\eta_{E} \eta_{B} G} \geq 0$. Pick $\epsilon=\frac{1}{2}\left(\sqrt{\eta_{E} \eta_{B} G} \mu_{\nu_{x}}+\theta-\sqrt{\eta_{E} \eta_{B} G} \alpha\right)>0$. So $-\theta+\sqrt{\eta_{E} \eta_{B} G} \alpha+\epsilon=\sqrt{\eta_{E} \eta_{B} G} \mu_{\nu_{x}}-\epsilon$. Then

$$
\begin{aligned}
& \int_{-\theta-\sqrt{\eta_{E} \eta_{B} G} \alpha}^{-\theta+\sqrt{\eta_{E} \eta_{B} G} \alpha} p_{N}(n) d n \\
& \leq \int_{-\infty}^{-\theta+\sqrt{\eta_{E} \eta_{B} G} \alpha} p_{N}(n) d n \\
& \leq \int_{-\infty}^{-\theta+\sqrt{\eta_{E} \eta_{B} G} \alpha+\epsilon} p_{N}(n) d n \\
& \leq \int_{-\infty}^{\sqrt{\eta_{E} \eta_{B} G} \mu_{\nu_{x}}-\epsilon} p_{N}(n) d n \\
& =\operatorname{Pr}\left\{N \leq \sqrt{\eta_{E} \eta_{B} G} \mu_{\nu_{x}}-\epsilon\right\} \\
& =\operatorname{Pr}\{N \leq \mu-\epsilon\} \\
& =\operatorname{Pr}\{N-\mu \leq-\epsilon\} \\
& \leq \operatorname{Pr}\{|N-\mu| \geq \epsilon\} \\
& \leq \frac{\sigma^{2}}{\epsilon^{2}}
\end{aligned}
$$

So the conditional probability difference in (44) vanishes as $\sigma_{\nu_{x}}^{2} \rightarrow 0$, as $r \rightarrow \infty$, as $\eta_{E}, \eta_{B} \rightarrow 1$, and as 
$G \rightarrow 1$ when $\mu_{\nu_{x}}>\theta+\alpha$. We lastly prove that the conditional probability difference in (45) vanishes as $\sigma_{\nu_{x}}^{2} \rightarrow 0$, as $r \rightarrow \infty$, as $\eta_{E}, \eta_{B} \rightarrow 1$, and as $G \rightarrow 1$ when $\mu_{\nu_{x}}>\theta+\alpha$. So $\sqrt{\eta_{E} \eta_{B} G}\left(\mu_{\nu_{x}}-\alpha\right)>\theta$ whenever $\sqrt{\eta_{E} \eta_{B} G}>\theta /\left(\mu_{\nu_{x}}-\alpha\right)$. Pick $\epsilon=\frac{1}{2}\left(\sqrt{\eta_{E} \eta_{B} G} \mu_{\nu_{x}}-\theta-\sqrt{\eta_{E} \eta_{B} G} \alpha\right)>0$. So $\theta+\sqrt{\eta_{E} \eta_{B} G} \alpha+\epsilon=\sqrt{\eta_{E} \eta_{B} G} \mu_{\nu_{x}}-\epsilon$. Then

$$
\begin{aligned}
& \int_{\theta-\sqrt{\eta_{E} \eta_{B} G} \alpha}^{\theta+\sqrt{\eta_{E} \eta_{B} G} \alpha} p_{N}(n) d n \\
& \leq \int_{-\infty}^{\theta+\sqrt{\eta_{E} \eta_{B} G} \alpha} p_{N}(n) d n \\
& \leq \int_{-\infty}^{\theta+\sqrt{\eta_{E} \eta_{B} G} \alpha+\epsilon} p_{N}(n) d n \\
& \leq \int_{-\infty}^{\sqrt{\eta_{E} \eta_{B} G} \mu_{\nu_{x}}-\epsilon} p_{N}(n) d n \\
& =\operatorname{Pr}\left\{N \leq \sqrt{\eta_{E} \eta_{B} G} \mu_{\nu_{x}}-\epsilon\right\} \\
& =\operatorname{Pr}\{N \leq \mu-\epsilon\} \\
& =\operatorname{Pr}\{N-\mu \leq-\epsilon\} \\
& \leq \operatorname{Pr}\{|N-\mu| \geq \epsilon\} \\
& \leq \frac{\sigma^{2}}{\epsilon^{2}}
\end{aligned}
$$

So the conditional probability difference in (45) as $\sigma_{\nu_{x}}^{2} \rightarrow$ 0 , as $r \rightarrow \infty$, and as $\eta_{E}, \eta_{B} \rightarrow 1$ when $\mu_{\nu_{x}}>\theta+\alpha$ and with the constraint $\sqrt{\eta_{E} \eta_{B} G}>\theta /\left(\mu_{\nu_{x}}-\alpha\right)$. Thus $\mu_{\nu_{x}} \notin(-\theta-\alpha,-\theta+\alpha) \cup(\theta-\alpha, \theta+\alpha)$ is a sufficient condition for the nonmonotone SR effect to occur with the given constraints on the product $\sqrt{\eta_{E} \eta_{B} G}$.

Proof (Necessity). We prove that the SR effect does not occur when $\mu_{\nu_{x}} \in(-\theta-\alpha,-\theta+\alpha) \cup(\theta-\alpha, \theta+\alpha)$.

Case 1: Suppose first that $\mu_{\nu_{x}} \in(-\theta-\alpha,-\theta+\alpha)$. We prove with a similar Chebyshev bound that the conditional probabilities $p_{Y \mid S}(0 \mid 0) \rightarrow 1$ and $p_{Y \mid S}(\varepsilon \mid 1) \rightarrow 1$ as $\sigma_{\nu_{x}}^{2} \rightarrow 0$, as $r \rightarrow \infty$, as $\eta_{E}, \eta_{B} \rightarrow 1$, and as $G \rightarrow 1$. Then the mutual information $I(S, Y)$ approaches its maximum $H(S)$ as all noise vanishes. Consider $p_{Y \mid S}(0 \mid 0)$. Pick any $\mu_{\nu_{x}} \in(-\theta-\alpha,-\theta+\alpha)$. Then $-\theta+\alpha>\mu_{\nu_{x}}$ and $\alpha-\mu_{\nu_{x}}>\theta$. Then $-\theta>$ $\sqrt{\eta_{E} \eta_{B} G}\left(\mu_{\nu_{x}}-\alpha\right)$ whenever $\sqrt{\eta_{E} \eta_{B} G}>\theta /\left|\alpha-\mu_{\nu_{x}}\right|$. Pick $\epsilon=\frac{1}{2}\left(-\theta+\sqrt{\eta_{E} \eta_{B} G} \alpha-\sqrt{\eta_{E} \eta_{B} G} \mu_{\nu_{x}}\right)>0$ so that
$-\theta+\sqrt{\eta_{E} \eta_{B} G} \alpha-\epsilon=\sqrt{\eta_{E} \eta_{B} G} \mu_{\nu_{x}}+\epsilon$.

$$
\begin{aligned}
p_{Y \mid S}(0 \mid 0) & \int_{-\infty}^{-\theta+\sqrt{\eta_{E} \eta_{B} G} \alpha} p_{N}(n) d n \\
\geq & \int_{-\infty}^{-\theta+\sqrt{\eta_{E} \eta_{B} G} \alpha-\epsilon} p_{N}(n) d n \\
= & \int_{-\infty}^{\sqrt{\eta_{E} \eta_{B} G} \mu_{\nu_{x}}+\epsilon} p_{N}(n) d n \\
= & 1-\int_{\sqrt{\eta_{E} \eta_{B} G} \mu_{\nu_{x}}+\epsilon}^{\infty} p_{N}(n) d n \\
= & 1-\operatorname{Pr}\left\{N \geq \sqrt{\eta_{E} \eta_{B} G} \mu_{\nu_{x}}+\epsilon\right\} \\
= & 1-\operatorname{Pr}\{N \geq \mu+\epsilon\} \\
= & 1-\operatorname{Pr}\{N-\mu \geq \epsilon\} \\
\geq & 1-\operatorname{Pr}\{|N-\mu| \geq \epsilon\} \\
\geq & 1-\frac{\sigma^{2}}{\epsilon^{2}} \\
\rightarrow & 1
\end{aligned}
$$

as $\sigma_{\nu_{x}}^{2} \rightarrow 0$, as $r \rightarrow \infty$, as $\eta_{E}, \eta_{B} \rightarrow 1$, and as $G \rightarrow 1$. We prove the result similarly for $p_{Y \mid S}(\varepsilon \mid 1)$. We show that $p_{Y \mid S}(0 \mid 1) \rightarrow 0$ and $p_{Y \mid S}(1 \mid 1) \rightarrow 0$ so that $p_{Y \mid S}(\varepsilon \mid 1) \rightarrow 1$. Pick any $\mu_{\nu_{x}} \in$ $(-\theta-\alpha,-\theta+\alpha)$. Then $\mu_{\nu_{x}}>-\theta-\alpha$ and $\mu_{\nu_{x}}+$ $\alpha>-\theta$. $\sqrt{\eta_{E} \eta_{B} G}\left(\mu_{\nu_{x}}+\alpha\right)>-\theta$ and $\sqrt{\eta_{E} \eta_{B} G} \mu_{\nu_{x}}+$ $\sqrt{\eta_{E} \eta_{B} G} \alpha+\theta>0$ whenever $\sqrt{\eta_{E} \eta_{B} G}<\theta /\left|\mu_{\nu_{x}}+\alpha\right|$. Pick $\epsilon=\frac{1}{2}\left(\sqrt{\eta_{E} \eta_{B} G} \mu_{\nu_{x}}+\sqrt{\eta_{E} \eta_{B} G} \alpha+\theta\right)>0$ so that $-\theta-\sqrt{\eta_{E} \eta_{B} G} \alpha+\epsilon=\sqrt{\eta_{E} \eta_{B} G} \mu_{\nu_{x}}-\epsilon$.

$$
\begin{aligned}
& p_{Y \mid S}(0 \mid 1) \\
& =\int_{-\infty}^{-\theta-\sqrt{\eta_{E} \eta_{B} G} \alpha} p_{N}(n) d n \\
& \leq \int_{-\infty}^{-\theta-\sqrt{\eta_{E} \eta_{B} G} \alpha+\epsilon} p_{N}(n) d n \\
& =\int_{-\infty}^{\sqrt{\eta_{E} \eta_{B} G} \mu_{\nu_{x}}-\epsilon} p_{N}(n) d n \\
& =\operatorname{Pr}\left\{N \leq \sqrt{\eta_{E} \eta_{B} G} \mu_{\nu_{x}}-\epsilon\right\} \\
& =\operatorname{Pr}\{N \leq \mu-\epsilon\} \\
& =\operatorname{Pr}\{N-\mu \leq-\epsilon\} \\
& \leq \operatorname{Pr}\{|N-\mu| \geq \epsilon\} \\
& \leq \frac{\sigma^{2}}{\epsilon^{2}}
\end{aligned}
$$

Pick any $\mu_{\nu_{x}} \in(-\theta-\alpha,-\theta+\alpha)$. Then $\mu_{\nu_{x}}<$ $-\theta+\alpha$ and $\theta<\alpha-\mu_{\nu_{x}} . \quad \sqrt{\eta G}\left(\mu_{\nu_{x}}-\alpha\right)<$ $-\theta$ and $-\sqrt{\eta_{E} \eta_{B} G} \mu_{\nu_{x}}+\sqrt{\eta_{E} \eta_{B} G} \alpha-\theta>0$ whenever $\sqrt{\eta_{E} \eta_{B} G}>\theta /\left|\alpha-\mu_{\nu_{x}}\right|$. Pick $\epsilon=$ $\frac{1}{2}\left(-\sqrt{\eta_{E} \eta_{B} G} \mu_{\nu_{x}}+\sqrt{\eta_{E} \eta_{B} G} \alpha-\theta\right)>0$ so that $-\theta+$ 


$$
\begin{aligned}
\sqrt{\eta_{E} \eta_{B} G} \alpha-\epsilon & =\sqrt{\eta_{E} \eta_{B} G} \mu_{\nu_{x}}+\epsilon . \\
p_{Y \mid S}(1 \mid 1) & p_{N}(n) d n \\
= & \int_{\theta-\sqrt{\eta_{E} \eta_{B} G \alpha}}^{\infty} p_{N}(n) d n \\
& \leq \int_{-\theta+\sqrt{\eta_{E} \eta_{B} G \alpha}}^{\infty} p_{N}(n) d n \\
& \leq \int_{-\theta+\sqrt{\eta_{E} \eta_{B} G} \alpha-\epsilon}^{\infty} p_{N}(n) d n \\
& =\int_{\sqrt{\eta_{E} \eta_{B} G} \mu_{\nu_{x}}+\epsilon}^{\infty} \\
& =\operatorname{Pr}\left\{N \geq \sqrt{\eta_{E} \eta_{B} G} \mu_{\nu_{x}}+\epsilon\right\} \\
& =\operatorname{Pr}\{N \geq \mu+\epsilon\} \\
& =\operatorname{Pr}\{N-\mu \geq \epsilon\} \\
& \leq \operatorname{Pr}\{|N-\mu| \geq \epsilon\} \\
& \leq \frac{\sigma^{2}}{\epsilon^{2}}
\end{aligned}
$$

So $p_{Y \mid S}(\varepsilon \mid 1) \rightarrow 1$ because $p_{Y \mid S}(0 \mid 1) \rightarrow 0$ and $p_{Y \mid S}(1 \mid 1) \rightarrow 0$ as $\sigma_{\nu_{r}}^{2} \rightarrow 0$, as $r \rightarrow \infty$, as $\eta_{E}, \eta_{B} \rightarrow 1$, and as $G \rightarrow 1$ and with constraint $\theta /\left|\alpha-\mu_{\nu_{x}}\right|<\sqrt{\eta_{E} \eta_{B} G}<\theta /\left|\alpha+\mu_{\nu_{x}}\right|$.

Case 2: Now suppose that $\mu_{\nu_{x}} \in(\theta-\alpha, \theta+\alpha)$. We prove that the conditional probabilities $p_{Y \mid S}(\varepsilon \mid 0) \rightarrow 1$ and $p_{Y \mid S}(1 \mid 1) \rightarrow 1$ as $\sigma_{\nu_{x}}^{2} \rightarrow 0$, as $r \rightarrow \infty$, as $\eta_{E}, \eta_{B} \rightarrow 1$, and as $G \rightarrow 1$. We first prove that $p_{Y \mid S}(\varepsilon \mid 0) \rightarrow 1$ in the limit of zero noise. We prove this by showing that $p_{Y \mid S}(0 \mid 0) \rightarrow 0$ and $p_{Y \mid S}(1 \mid 0) \rightarrow 0$ in the limit. Pick any $\mu_{\nu_{x}} \in(\theta-\alpha, \theta+\alpha)$. Then $\mu_{\nu_{x}}>\theta-\alpha$ and $\mu_{\nu_{x}}+\alpha>\theta . \sqrt{\eta_{E} \eta_{B} G}\left(\mu_{\nu_{x}}+\alpha\right)>\theta$ and $\sqrt{\eta_{E} \eta_{B} G} \mu_{\nu_{x}}+$ $\sqrt{\eta_{E} \eta_{B} G} \alpha-\theta>0$ whenever $\sqrt{\eta_{E} \eta_{B} G}>\theta /\left(\mu_{\nu_{x}}+\alpha\right)$. Pick $\epsilon=\frac{1}{2}\left(\sqrt{\eta_{E} \eta_{B} G} \mu_{\nu_{x}}+\sqrt{\eta_{E} \eta_{B} G} \alpha-\theta\right)>0$ so that $\theta-\sqrt{\eta_{E} \eta_{B} G} \alpha+\epsilon=\sqrt{\eta_{E} \eta_{B} G} \mu_{\nu_{x}}-\epsilon$.

$$
\begin{aligned}
& p_{Y \mid S}(0 \mid 0) \\
& =\int_{-\infty}^{-\theta+\sqrt{\eta_{E} \eta_{B} G} \alpha} p_{N}(n) d n \\
& \leq \int_{-\infty}^{\theta-\sqrt{\eta_{E} \eta_{B} G} \alpha} p_{N}(n) d n \\
& \leq \int_{-\infty \quad p_{N}(n) d n}^{\theta-\sqrt{\eta_{E} \eta_{B} G} \alpha+\epsilon} p_{N}(n) d n \\
& =\int_{-\infty}^{\sqrt{\eta_{E} \eta_{B} G} \mu_{\nu_{x}}-\epsilon} \\
& =\operatorname{Pr}\left\{N \leq \sqrt{\eta_{E} \eta_{B} G} \mu_{\nu_{x}}-\epsilon\right\} \\
& =\operatorname{Pr}\{N \leq \mu-\epsilon\} \\
& =\operatorname{Pr}\{N-\mu \leq-\epsilon\} \\
& \leq \operatorname{Pr}\{|N-\mu| \geq \epsilon\} \\
& \leq \frac{\sigma^{2}}{\epsilon^{2}}
\end{aligned}
$$

Pick any $\mu_{\nu_{x}} \in(\theta-\alpha, \theta+\alpha)$. Then $\mu_{\nu_{x}}<\theta+\alpha$ and $\mu_{\nu_{x}}-\alpha<\theta . \quad \sqrt{\eta_{E} \eta_{B} G}\left(\mu_{\nu_{x}}-\alpha\right)<\theta$ and $-\sqrt{\eta_{E} \eta_{B} G} \mu_{\nu_{x}}+\sqrt{\eta_{E} \eta_{B} G} \alpha+\theta>0$ whenever $\sqrt{\eta_{E} \eta_{B} G}<\theta /\left|\mu_{\nu_{x}}-\alpha\right|$. Pick $\epsilon=$ $\frac{1}{2}\left(-\sqrt{\eta_{E} \eta_{B} G} \mu_{\nu_{x}}+\sqrt{\eta_{E} \eta_{B} G} \alpha+\theta\right)>0$ so that $\theta+\sqrt{\eta_{E} \eta_{B} G} \alpha-\epsilon=\sqrt{\eta_{E} \eta_{B} G} \mu_{\nu_{x}}+\epsilon$.

$$
\begin{aligned}
& p_{Y \mid S}(1 \mid 0) \\
& =\int_{\theta+\sqrt{\eta_{E} \eta_{B} G \alpha}}^{\infty} p_{N}(n) d n \\
& \leq \int_{\theta+\sqrt{\eta_{E} \eta_{B} G} \alpha-\epsilon}^{\infty} p_{N}(n) d n \\
& =\int_{\sqrt{\eta_{E} \eta_{B} G} \mu_{\nu_{x}}+\epsilon}^{\infty} p_{N}(n) d n \\
& =\operatorname{Pr}\left\{N \geq \sqrt{\eta_{E} \eta_{B} G} \mu_{\nu_{x}}+\epsilon\right\} \\
& =\operatorname{Pr}\{N \geq \mu+\epsilon\} \\
& =\operatorname{Pr}\{N-\mu \geq \epsilon\} \\
& \leq \operatorname{Pr}\{|N-\mu| \geq \epsilon\} \\
& \leq \frac{\sigma^{2}}{\epsilon^{2}}
\end{aligned}
$$

So $p_{Y \mid S}(\varepsilon \mid 0) \rightarrow 1$ because $p_{Y \mid S}(0 \mid 0) \rightarrow 0$ and $p_{Y \mid S}(1 \mid 0) \rightarrow$ 0 as $\sigma_{\nu_{x}}^{2} \rightarrow 0$, as $r \rightarrow \infty$, as $\eta_{E}, \eta_{B} \rightarrow 1$, and as $G \rightarrow 1$. Now we prove that $p_{Y \mid S}(1 \mid 1) \rightarrow 1$ as $\sigma_{\nu_{x}}^{2} \rightarrow 0$, as $r \rightarrow \infty$, as $\eta_{E}, \eta_{B} \rightarrow 1$, and as $G \rightarrow 1$ whenever $\mu_{\nu_{x}} \in(\theta-\alpha, \theta+\alpha)$. Pick any $\mu_{\nu_{x}} \in(\theta-\alpha, \theta+\alpha)$. Then $\mu_{\nu_{x}}<\theta+\alpha$ and $\mu_{\nu_{x}}-$ $\alpha<\theta . \quad \sqrt{\eta_{E} \eta_{B} G}\left(\mu_{\nu_{x}}-\alpha\right)<\theta$ and $-\sqrt{\eta_{E} \eta_{B} G} \mu_{\nu_{x}}+$ $\sqrt{\eta_{E} \eta_{B} G} \alpha+\theta>0$ whenever $\sqrt{\eta_{E} \eta_{B} G}<\theta /\left|\mu_{\nu_{x}}-\alpha\right|$. Pick $\epsilon=\frac{1}{2}\left(-\sqrt{\eta_{E} \eta_{B} G} \mu_{\nu_{x}}+\sqrt{\eta_{E} \eta_{B} G} \alpha+\theta\right)>0$ so that $\theta-\sqrt{\eta_{E} \eta_{B} G} \alpha+\epsilon=\sqrt{\eta_{E} \eta_{B} G} \mu_{\nu_{x}}-\epsilon$.

$$
\begin{aligned}
& p_{Y \mid S}(1 \mid 1) \\
& =\int_{\theta-\sqrt{\eta_{E} \eta_{B} G \alpha}}^{\infty} p_{N}(n) d n \\
& \geq \int_{\theta-\sqrt{\eta_{E} \eta_{B} G} \alpha+\epsilon}^{\infty} p_{N}(n) d n \\
& =\int_{\sqrt{\eta_{E} \eta_{B} G} \mu_{\nu_{x}}-\epsilon}^{\infty} p_{N}(n) d n \\
& =1-\int_{-\infty}^{\sqrt{\eta_{E} \eta_{B} G} \mu_{\nu_{x}}-\epsilon} p_{N}(n) d n \\
& =1-\operatorname{Pr}\left\{N \leq \sqrt{\eta_{E} \eta_{B} G} \mu_{\nu_{x}}-\epsilon\right\} \\
& =1-\operatorname{Pr}\{N \leq \mu-\epsilon\} \\
& =1-\operatorname{Pr}\{N-\mu \leq-\epsilon\} \\
& \geq 1-\operatorname{Pr}\{|N-\mu| \geq \epsilon\} \\
& \geq 1-\frac{\sigma^{2}}{\epsilon^{2}}
\end{aligned}
$$

So $p_{Y \mid S}(1 \mid 1) \rightarrow 1$ as $\sigma_{\nu_{x}}^{2} \rightarrow 0$, as $r \rightarrow \infty$, as $\eta_{E}, \eta_{B} \rightarrow$ 1 , and as $G \rightarrow 1$ whenever $\mu_{\nu_{x}} \in(\theta-\alpha, \theta+\alpha)$ and with constraint $\theta /\left(\mu_{\nu_{x}}+\alpha\right)<\sqrt{\eta_{E} \eta_{B} G}<\theta /\left|\mu_{\nu_{x}}-\alpha\right|$. 
The mutual information $I(S, Y)$ approaches its maximum $H(S)$ as all noise vanishes and the SR effect does not occur for Alice and Bob's mutual information whenever $\mu_{\nu_{x}} \in(-\theta-\alpha,-\theta+\alpha) \cup(\theta-\alpha, \theta+\alpha)$ with the above constraints on the product $\sqrt{\eta_{E} \eta_{B} G}$.

\section{Proof of Theorem 2 (Infinite Variance)}

The proof for sufficiency and necessity follows the same stable proof method with some modifications. We use the same characteristic function $\varphi_{\nu_{x}}(\omega)$ in (18) for alphastable random variable $\nu_{x}$. Suppose

$$
\sigma_{\mathcal{N}}^{2}=\left(\eta_{B}\left(\begin{array}{c}
\eta_{E} G e^{-2 r}+\eta_{E}(G-1) \\
+\left(1-\eta_{E}\right)
\end{array}\right)+1-\eta_{B}\right) / 2
$$

The characteristic function $\varphi_{N}(\omega)$ of $p_{N}(n)$ is as follows

$$
\varphi_{N}(\omega)=\varphi_{\nu_{x}}\left(\sqrt{\eta_{E} \eta_{B} G} \omega\right) \exp \left\{-\frac{\sigma_{\mathcal{N}}^{2} \omega^{2}}{2}\right\}
$$

from (5) and the convolution theorem.

Proof (Sufficiency). Take the limit of the characteristic function $\varphi_{N}(\omega)$ as $\gamma \rightarrow 0$, as $r \rightarrow \infty$, as $G \rightarrow 1$, and as $\eta_{E}, \eta_{B} \rightarrow 1$ to obtain the following characteristic function.

$$
\lim _{\substack{r \rightarrow \infty, \gamma \rightarrow 0, G \rightarrow 1, \eta_{E}, \eta_{B} \rightarrow 1}} \varphi_{N}(\omega)=\exp \{i a \omega\}
$$

The probability density $p_{N}(n)$ then approaches a translated delta function

$$
\lim _{r \rightarrow \infty, \gamma \rightarrow 0, \eta_{E}, \eta_{B} \rightarrow 1} p_{N}(n)=\delta(n-a)
$$

Suppose that $a \notin(-\theta-\alpha,-\theta+\alpha) \cup(\theta-\alpha, \theta+\alpha)$. Consider the case where $y=0$. Then

$$
\begin{aligned}
& \lim _{\substack{r \rightarrow \infty, \gamma \rightarrow 0, G \rightarrow 1, \eta_{E}, \eta_{B} \rightarrow 1}} p_{Y \mid S}(0 \mid 0)-p_{Y \mid S}(0 \mid 1) \\
= & \lim _{\substack{r \rightarrow \infty, \gamma \rightarrow 0, G \rightarrow 1, \eta_{E}, \eta_{B} \rightarrow 1}} \int_{-\theta-\sqrt{\eta_{E} \eta_{B} G} \alpha}^{-\theta+\sqrt{\eta_{E} \eta_{B} G} \alpha} p_{N}(n) d n \\
\rightarrow & \int_{-\theta-\alpha}^{-\theta+\alpha} \delta(n-a) d n=0
\end{aligned}
$$

because $a \notin(-\theta-\alpha,-\theta+\alpha)$. Consider the case where $y=1$.

$$
\begin{aligned}
& \lim _{\substack{r \rightarrow \infty, \gamma \rightarrow 0, G \rightarrow 1, \eta_{E}, \eta_{B} \rightarrow 1}} p_{Y \mid S}(1 \mid 1)-p_{Y \mid S}(1 \mid 0) \\
= & \lim _{\substack{r \rightarrow \infty, \gamma \rightarrow 0, G \rightarrow 1, \eta_{E}, \eta_{B} \rightarrow 1}} \int_{\theta-\sqrt{\eta_{E} \eta_{B} G} \alpha}^{\theta+\sqrt{\eta_{E} \eta_{B} G} \alpha} p_{N}(n) d n \\
\rightarrow & \int_{\theta-\alpha}^{\theta+\alpha} \delta(n-a) d n=0
\end{aligned}
$$

because $a \notin(\theta-\alpha, \theta+\alpha)$.

Proof (Necessity). Suppose that $a \in$ $(-\theta-\alpha,-\theta+\alpha) \cup(\theta-\alpha, \theta+\alpha)$.

Case 1: Pick $a \in(-\theta-\alpha,-\theta+\alpha)$. We show that 
$p_{Y \mid S}(0 \mid 0) \rightarrow 1$ and $p_{Y \mid S}(\varepsilon \mid 1) \rightarrow 1$. Then

$$
\begin{aligned}
p_{Y \mid S}(0 \mid 0) & =\int_{-\infty}^{-\theta+\sqrt{\eta_{E} \eta_{B} G} \alpha_{x}} p_{N}(n) d n \\
& =\int_{-\infty}^{-\theta} p_{N}\left(n+\sqrt{\eta_{E} \eta_{B} G} \alpha_{x}\right) d n \\
& \rightarrow \int_{-\infty}^{-\theta} \delta(n-a+\alpha) d n \\
& =\int_{-\infty}^{-\theta+\alpha} \delta(n-a) d n=1 \\
p_{Y \mid S}(\varepsilon \mid 1) & =\int_{-\theta-\sqrt{\eta_{E} \eta_{B} G} \alpha}^{\theta-\sqrt{\eta_{E} \eta_{B} G} \alpha} p_{N}(n) d n \\
\rightarrow & \int_{-\theta-\alpha}^{\theta-\alpha} \delta(n-a) d n \\
& =1
\end{aligned}
$$

Case 2: Pick $a \in(\theta-\alpha, \theta+\alpha)$. We show that

$p_{Y \mid S}(1 \mid 1) \rightarrow 1$ and $p_{Y \mid S}(\varepsilon \mid 0) \rightarrow 1$. Then

$$
\begin{aligned}
& p_{Y \mid S}(1 \mid 1)=\int_{\theta-\sqrt{\eta_{E} \eta_{B} G} \alpha}^{\infty} p_{N}(n) d n \\
&=\int_{\theta}^{\infty} p_{N}\left(n-\sqrt{\eta_{E} \eta_{B} G} \alpha_{x}\right) d n \\
& \rightarrow \int_{\theta}^{\infty} \delta(n-a-\alpha) d n \\
&=\int_{\theta-\alpha}^{\infty} \delta(n-a) d n=1 \\
& p_{Y \mid S}(\varepsilon \mid 0)=\int_{-\theta+\sqrt{\eta_{E} \eta_{B} G} \alpha}^{\theta+\sqrt{\eta_{E} \eta_{B} G} \alpha} p_{N}(n) d n \\
& \rightarrow \int_{-\theta+\alpha}^{\theta+\alpha} \delta(n-a) d n \\
&=1
\end{aligned}
$$

[1] R. Benzi et al., Journal of Physics A 14, 453 (1981).

[2] K. Wiesenfeld and F. Moss, Nature 373, 33 (1995).

[3] A. R. Bulsara and L. Gammaitoni, Physics Today 49, 39 (1996).

[4] L. Gammaitoni et al., Rev. Mod. Phys. 70, 223 (1998).

[5] B. Kosko, Noise (Viking/Penguin, 2006).

[6] I. Goychuk and P. Hänggi, New J. Phys. 1, 14 (1999).

[7] B. Kosko and S. Mitaim, Neural Networks 14, 755 (2003).

[8] B. Kosko and S. Mitaim, Phys. Rev. E 70, 031911 (2004).

[9] B. Kosko and S. Mitaim, Phys. Rev. E 64, 051110 (2001).

[10] R. Loudon and P. L. Knight, Modern Optics 34, 709 (1987).

[11] C. C. Gerry and P. L. Knight, Introductory Quantum Optics (Cambridge University Press, 2005).

[12] T. Hirano et al., quant-ph/0008037 (2000).

[13] R. Namiki and T. Hirano, Phys. Rev. A 67, 022308 (2003).

[14] A. S. Holevo and R. F. Werner, Phys. Rev. A 63, 032312 (2001).

[15] T. M. Cover and J. A. Thomas, Elements of Information
Theory (Wiley-Interscience, 1991).

[16] U. Leonhardt and H. Paul, Phys. Rev. A 48, 4598 (1993).

[17] M. J. W. Hall, Phys. Rev. A 50, 3295 (1994).

[18] R. J. Glauber, in Les Prix Nobel. The Nobel Prizes 2005, edited by K. Grandin (Nobel Foundation, 2005), pp. 9091.

[19] L. Breiman, Probability (Addison-Wesley, 1968).

[20] C. Nikias and M. Shao, Signal Processing with AlphaStable Distributions and Applications (John Wiley and Sons, 1995).

[21] U. M. Maurer, IEEE Trans. Inf. Theory 39, 733 (1993).

[22] R. Namiki and T. Hirano, Phys. Rev. A 72, 024301 (2005).

[23] H. A. Haus and J. A. Mullen, Phys. Rev. 128, 2407 (1962).

[24] M. A. Nielsen and I. L. Chuang, Quantum Computation and Quantum Information (Cambridge University Press, 2000).

[25] I. Devetak, IEEE Trans. Inf. Theory 51, 44 (2005). 